\title{
Fuzzy Entropy based Impulse Noise Detection and Correction Method for Digital Images
}

\author{
S.Vijaya Kumar \\ Research Scholar, Dept of CSE, JNTU Hyderabad, Telangana, \\ Email: svksr105@gmail.com \\ C.Nagaraju \\ Associate Professor, \\ YSR Engineering College of Yogivemana University, Proddatur, A.P \\ Email: cnrcse@yahoo.com
}

Received: 08 December 2017; Accepted: 30 January 2018; Published: 08 March 2018

\begin{abstract}
Impulse noise is the prime factor which reduces the quality of the digital image and it erases the important details of the images. De-noising is an indispensable task to restore the image features from the corrupted low- quality images and improve the perceptual quality of images. Several techniques are used for image quality enhancement and image restoration. In this work, an image de-noising scheme is developed to detect and correct the impulse noise from the image by using fuzzy entropy. The proposed algorithm is designed in two phases, such as noise detection phase, and correction phase. In the noise detection phase, the fuzzy entropy of pixels in a window of interest (WoI) is computed to detect whether the pixel is noisy or not. The Fuzzy entropy of pixel greater than specified alpha cut value will be considered as noise pixel and submitted to correction phase. In the correction phase noise pixel value is replaced with a fuzzy weighted mean of the uncorrupted pixels in the WoI. The proposed Fuzzy entropy based impulse noise detection and correction method are implemented using MATLAB. The experimentation has been carried out on different standard images and the analysis is performed by comparing the performance of the proposed scheme with that of the existing methods such as DBA, MDBUTMF, AMF, NAFSM, BDND, and CM , using PSNR, SSIM, and NAE as metric parameters. The proposed method will give good results compared to state of the art methods in image restoration.
\end{abstract}

Index Terms-Fuzzy entropy, a window of interest, impulse noise, image restoration.

\section{INTRODUCTION}

With the advent and usage of multimedia, visual data from quality digital images play a significant role in human day to day life applications. Unfortunately, images which are captured through many cameras have been generally subjected to the contamination of impulse noise. Generally impulse noise caused by malfunctioning pixel sensors, defective memory units, and imperfections encountered in a channel for the duration of transmission and timing errors in analog-to-digital conversion [1-4]. Before successive digital image processing operations, restoring of the corrupted image has been accomplished as the first step in digital image processing.

Image restoration is imperative for successive tasks (e.g., edge detection, image segmentation, classification, parameter estimation, etc.) which are basically affected by the quality of the image. Capturing devices has become sensitive to the acquaintance of impulse noise due to more sensing elements per unit space are integrated on a single chip. To overcome this, digital camera manufacturing companies rely on restoration methods to enhance the visual quality of the image acquired. As a result, a number of methods have been proposed for the removal of impulse noise. Non-linear filters are superior to linear filter with their great execution to restoring the image from impulse noise, For instance, the median filter [2],[5] could be a natural selection for suppressing impulse noise. The idea of a median filter is to replace the window pixel given by the median of the brightness in the window. The Median filter gives the better results at low noise levels $(<10 \%)[12]$ but it alters the image pixels even though it is not corrupted, this led to bad result at high noise levels $(>10 \%)[12]$, the key image details are also decorated. This problem has led to the development of various classes of filters, such as the weighted median-filters [1], [2], [4]-[7], [9], adaptive filters [8],[12], and rank-ordered statistics, switching median filter and soft computing filters [9-14],[16], [28-29].By adapting these non-linear filters, the restoration quality Significantly increases but the implementation and time complexity is multiplied and hardware cost also increases. In this paper, we propose a new filtering mechanism is proposed for high impulse noise removal with less computational cost using fuzzy entropy. The proposed technique restored the digital image with less computational time and it simultaneously maintaining edge information compare with different existing filters. 


\section{IMPULSE NOISE MODELS}

In this section, impulse noise models are clearly described. A digital image of size $\mathrm{RxC}$ stored as an 8-bit gray level image and the image elements are lie in the range $[0,255]$. In this, least and highest intensity values are 0 and 255. Regardless of its origin, impulse noise shows non stationary measurable qualities [1], [3] and just a specific percentage of pixels in the digital images are corrupted by impulse noise [12]. In view of this reality, the models for impulse noise with probability $\mathrm{P}$ defined in[12] as

$$
S N P_{-} \operatorname{Image}(i, j)=\left\{\begin{array}{l}
\frac{P}{2}, \text { for } \operatorname{In} \_\operatorname{Image}(i, j)=0 \\
1-P, \text { for In_Image }(i, j) \\
\frac{P}{2}, \text { for } \operatorname{In}{ }_{-} \operatorname{Image}(i, j)=255
\end{array}\right.
$$

where the $\mathrm{P}$ is the noise probability density

\section{Noise Model-I}

$$
S N P \_ \text {Image }(i, j)=\left\{\begin{array}{l}
P 1, \text { for In_Image }(i, j)=0 \\
1-P, \text { for In_Image }(i, j) \\
P 2, \text { for In_Image }(i, j)=255
\end{array}\right.
$$

where the $\mathrm{P}$ is the noise probability density $\mathrm{P}=\mathrm{P} 1+\mathrm{P} 2$ and $\mathrm{P} 1 \neq \mathrm{P} 2$

\section{Noise Model-II}

In the literature, usually two impulse noise models: salt-and-pepper (SNP) noise and random-valued impulse noise. Which are used as a part of image processing. In the SNP noise the image intensity values are set to be 255 (salt) that is all the bits in gray level set to be one and 0 (pepper) that is all the bits in gray level set to be zeros. In random-valued impulse noise model, the image intensity values are set to be any value within the dynamic range $[0,255]$.In the real world scenario, impulse noise is generated from the overlapping of impulse noise signals with random amplitudes. As a result, the impulsive amplitude could both fall within the image dynamic range or out of that range. While the impulsive amplitude lies outside of the dynamic range, the resultant pixel might be saturated and threshold to the 255 or 0 intensity value of the image and looks as SNP noise. On the other hand, if the impulsive amplitude lies in the dynamic range, the resultant pixel seems as uniform noise (UNIF) noise within the image.

The noise model-I is SNP model with equal probability as represented in equation-1 and Noise Model II is similar to model-II, except that salt noise and pepper noise are with unequal probability as represented in equation- 2 .

$$
\text { Unif_Image }(i, j)=\left\{\begin{array}{l}
\frac{P}{2 M}, \text { for } 0 \leq \text { In_Image }(i, j)<M \\
1-P, \text { for In_Image }(i, j) \\
\frac{P}{2 M}, \text { for } 255-M<\text { In_Image }(i, j) \leq 255
\end{array}\right.
$$

where the $\mathrm{P}$ is the noise probability density

\section{Noise Model-III}

Unif_Image $(i, j)=\left\{\begin{array}{l}\frac{P 1}{M}, \text { for } 0 \leq \operatorname{In} \_\operatorname{Im} a g e(i, j)<M \\ 1-P, \text { for } \operatorname{In} \_\operatorname{Im} a g e(i, j) \\ \frac{P 2}{M}, \text { for } 255-M<\operatorname{In} \_\operatorname{Im} \operatorname{age}(i, j) \leq 255\end{array}\right.$

where the $\mathrm{P}$ is the noise probability density $\mathrm{P}=\mathrm{P} 1+\mathrm{P} 2$ and $\mathrm{P} 1 \neq \mathrm{P} 2$

\section{Noise Model-IV}

The noise model-III is UNIF model with equal probability as represented in equation-3 and Noise Model IV is similar to model III, except that salt noise and pepper noise are with unequal probability as represented in equation-4.

\section{FUZZY ENTROPY MEASURE FOR IMPULSE NOISE IDENTIFICATIONS}

An image $\mathrm{X}$ of size $\mathrm{RxC}$ having $\mathrm{L}$ gray levels ranging from $\mathrm{L}_{\min }$ to $\mathrm{L}_{\max }$ can be defined as an array of fuzzy singletons .Each element in the array is the membership function value representing its degree of brightness relative to gray level $l\left(l=\mathrm{L}_{\min }, \mathrm{L}_{\min +1}, \ldots, \mathrm{L}_{\max }\right)$. Therefore, in fuzzy set notation, we can write

$$
\begin{gathered}
X=\{\mu p(i, j) / p(i, j), i=1,2,3,4, \ldots \ldots \ldots, R ; j=1,2,3,4, \ldots . ., C\} \\
\mu p_{(i, j)}=e^{-\frac{\left(p_{(i, j)}-\bar{P}\right)^{2}}{2 \sigma^{2}}}
\end{gathered}
$$

Where

$$
\sigma=\sqrt{\frac{\pi}{2}} \times \frac{1}{m n} \sum_{i=1}^{m} \sum_{j=1}^{n}|p(i, j)-\bar{P}|
$$

Where

$$
\bar{P}=\frac{1}{m n} \sum_{i=1}^{m} \sum_{j=1}^{n} p(i, j)
$$

From the entropy concept, we know that fuzzy entropy [17-22],[27] is less for orderly image pixel values and more for disorderly image pixels. If we try to visualize the image data, information pixels are orderly configured and impulse noise pixels are disorderly configured. So if we evaluate the fuzzy entropy at each image pixel then the image pixel with minimum fuzzy entropy is an informative pixel and with higher fuzzy entropy is an impulse noise pixel. 
The fuzzy entropy value of pixel in given a window of interest can be calculated as given in equation- 5

$$
\begin{aligned}
& \mathrm{E}\left(\mu\left(p_{i}\right)\right)=-\mu\left(p_{i}\right) \log 2 \mu\left(p_{i}\right)- \\
& \left(1-\mu\left(p_{i}\right)\right) \log 2\left(1-\mu\left(p_{i}\right)\right), 0 \leq \mu\left(p_{i}\right) \leq 1
\end{aligned}
$$

The fuzzy entropy value of the image pixels in the given window is in the range [0.0-1.0].Fuzzy Entropy Value is low for the pixel, which lie between 0 and 255 and very high (close to 1) for the 0 and 255. Fuzzy entropy assumes the maximum value of 1.0 when $\mu\left(p_{(i, j)}\right)$ is 0.5 and a minimum value of 0.0 when $\mu\left(p_{(i, j)}\right)$ is 0.0 or $1.0[22]$.

\section{IMPUlSE NoISE DETECTION METHOD USING FuZZY ENTROPY MEASURE}

This section describes the Fuzzy Entropy Based Impulse Noise Detection (FEIND) algorithm and then discusses the some of the implementation issues of the algorithm. The algorithm-I is used to detect the pixel is impulsive or not with fuzzy entropy. The basic working principle of an algorithm-I is that, given image convert into the fuzzy plane using the Gaussian membership function, then compute the fuzzy entropy of image pixels using the equation-5.

A greater difference of the values among the evaluation pixels results in a higher fuzzy entropy and pixel values which are similar in the window results in lower fuzzy entropy. Using this underlying idea the pixels can be classified as corrupted or uncorrupted. To identify given pixel in the window of interest (WoI) is impulsive or not, calculate the fuzzy entropy of processing pixel in the WoI and check whether the fuzzy entropy of pixel is greater than the given alpha cut value. If it is greater than the given alpha cut value then it is impulsive and submitted to correction phase. Skelton of the noise detection is given in algorithm-I.

\section{A. Impulse Noise Detection Method Using Fuzzy Entropy Measure}

FEIND method keeps its original value as it is in the processed image if it detects the pixel values is informative. Only impulse noise pixels are submitted to correction phase, which is similar to traditional switching filters. Traditional switching filters first identify the noise pixels and form the binary noise map of the image to record the information of impulse noise such as noise pixel location. Fuzzy weighted mean filter corrects the noise pixel one after another using the information in noise map and it leads to increase of CPU time and require more main memory space. To overcome this drawback, the FEIND filter corrects the impulse noise immediately after the pixel has been detected as an impulsive candidate. Hence, in the FEIND filter, for the noise detection and the correction use the same window of interest. The details are shown in the algorithm-I.

\section{RESUlTS AND DisCUSSIONS}

In this section, from the experimental study we are going to address the following two points:

1. FEIND method can identify the impulse noise pixels in given image

2. Restore the corrupted pixel value with the fuzzy mean of uncorrupted pixels in WoI.

In order to accomplish the tasks, we use Lena, Parrot, and bridge corrupted mages with impulse noise model-I, noise density range from $30 \%$ to $90 \%$. To show FEIND is able to detect the impulse noise candidate, we experimented with different window size on various noise levels by choosing the different alpha cut values range from 0.1 to 0.9 . The Fig. 1 Depicts the input $7 \times 7$ image with $70 \%$ of impulse noise and Figure. 2 represent the fuzzy entropy of the corrupted image. From the Table-I we can conclude that the pixels with intensities 0 and 255 having greater fuzzy entropy than the remaining pixels. The pixel is treated as a noise pixel if it having greater fuzzy entropy than the specified alpha cut value and it is submitted to next filtering stage. Otherwise, the pixel is treated as informative pixel and it keeps as it is in the processed image. Table IV, Table V, Table VI and Table VII lists the accuracies of identification of the noise pixels with various window sizes, which are defined using two factors. One is the number of missed detections (MD), namely, the number of noise pixels that are identified as uncorrupted pixels. The other one is the number of false alarms (FA), i.e., the number of uncorrupted pixels that are identified as noise pixels [12]. Among the filtering windows, the $7 \times 7$ has less MD and

\section{Algorithm-I}

For every pixel $\mathrm{p}_{\mathrm{i}, \mathrm{j}}$ image do

1. Get region $R$ for image pixel $p_{i, j}$ by taking $M=4$

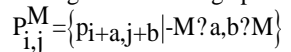

2. Calculate the sample mean of the Region p, i.e.,

$p\urcorner \frac{1}{\mathrm{mn}} \sum_{\mathrm{i}=1}^{\mathrm{m}} \sum_{\mathrm{j}=1}^{\mathrm{n}} \mathrm{p}(\mathrm{i}, \mathrm{j})$

3. Calculate the Standard Deviation of the Region $\mathrm{p}$, i.e.,

$$
\sigma \neg \sqrt{\frac{\pi}{2}} \times \frac{1}{m n} \sum_{i=1}^{m} \sum_{j=1}^{n}|p(i, j)-\bar{P}|
$$

4. Calculate the fuzzy membership of the processing pixel $p_{i, j}$, i.e.

$$
\mu\left(p_{i, j}\right)=e^{-\frac{\left(p_{i, j}-\bar{P}\right)^{2}}{2 \sigma^{2}}}
$$

5. Calculate the fuzzy entropy of the processing pixel $\mathrm{p}_{\mathrm{i}, \mathrm{j}}$, i.e.,

$E\left(\mu\left(p_{i, j}\right)\right)=-\mu\left(p_{i, j}\right) \log 2 \mu\left(p_{i, j}\right)-\left(1-\mu\left(p_{i, j}\right)\right) \log 2\left(1-\mu\left(p_{i, j}\right)\right), 0 \leq \mu\left(p_{i, j}\right) \leq 1$

6. $\operatorname{ifE}\left(\mu\left(\mathrm{p}_{i, j}\right)\right)<\mathrm{T}$ then

$$
\mathrm{p}_{\mathrm{i}, \mathrm{j}}=\mathrm{p}_{\mathrm{i}, \mathrm{j}} \text {, continue }
$$

7. else

8. $\mathrm{p}_{\mathrm{i}, \mathrm{j}}$ is corrupted with impulse noise 


\section{Algorithm-II}

1. Calculate the sample mean of the pixels in the Region $p$, i.e., $\overline{\mathrm{p}} \neg \frac{1}{\mathrm{mn}} \sum_{\mathrm{i}=1}^{\mathrm{m}} \sum_{\mathrm{j}=1}^{\mathrm{n}} \mathrm{p}(\mathrm{i}, \mathrm{j})$

2. Calculate the Standard Deviation i.e.,

$$
\sigma \neg \sqrt{\frac{\pi}{2}} \times \frac{1}{m n} \sum_{i=1}^{m} \sum_{j=1}^{n}|p(i, j)-\bar{P}|
$$

3. Calculate the fuzzy membership of the pixels in $t p_{i, j}$, i.e.,

$$
\mu_{\mathrm{F}}\left(\mathrm{p}_{\mathrm{i}}\right)=\mathrm{e}^{-\frac{\left(\mathrm{p}_{\mathrm{i}, \mathrm{j}}-\overline{\mathrm{P}}\right)^{2}}{2 \sigma^{2}}}
$$

4. Calculate the fuzzy entropy of the processing pixel $\mathrm{p}_{\mathrm{i}, \mathrm{j}}$, i.e.,

$E\left(\mu\left(p_{i, j}\right)\right)=-\mu\left(p_{i, j}\right) \log 2 \mu\left(p_{i, j}\right)-\left(1-\mu\left(p_{i, j}\right)\right) \log 2\left(1-\mu\left(p_{i, j}\right)\right), 0 \leq \mu\left(p_{i, j}\right) \leq 1$

5. $\operatorname{ifE}\left(\mu\left(\mathrm{p}_{\mathrm{i}, \mathrm{j}}\right)\right)<\mathrm{T}$ then

fuzzy weights of uncorrupted pixel is $w_{i}=\mu \quad\left(p_{i, j}\right)=e^{-\frac{\left(p_{i, j}-\bar{P}\right)^{2}}{2 \sigma^{2}}}$

6.compute the fuzzy weighted mean of uncorrupted pixel, i.e.,

$\mathrm{y}_{\mathrm{i}, \mathrm{j}}=\sum_{\mathrm{i}=1}^{\mathrm{n}} \mathrm{p}_{\mathrm{i}}^{*} \mu\left(\mathrm{p}_{\mathrm{i}}\right)$

where $n=$ number of un-corrupted pixels in the region of interest

FA (see Table-III\&VIII) at the alpha cut value in between 0.5 to 0.6 . Therefore, image restoration using $7 \times 7$ window gives the better visual quality than the remaining windows. All the windows up to 0.4 alpha cut value gives the zero false alarms but gives more missed detections. For the $60 \%$ and $80 \%$ impulse noise, the window size $5 \times 5$ with alpha cut vale 0.6 gives the least missed detection and zero false alarms. The window size $7 \times 7$ with alpha cut vale 0.5 gives the least missed detections and zero false alarms for the noise densities range from 30 to 90.The window size 9x9 with alpha cut value 0.6 gives the least missed detections and zero false alarms for the noise density $60 \%$ and gives the zero missed detection and least false alarms for the noise density $70 \%$.From the Tables IV to VII finally concluded that the alpha cut value between 0.5 to 0.6 with window size $7 \times 7$ gives least missed detections and false alarms. Table-IX shows the identification time required to classify the noisy pixel and information pixel using various windows. From the above analysis we concluded that the window size $5 \times 5$ gives least computational time and $11 \times 11$ gives the more computational time. From the table-VII, For the $90 \%$ of impulse noise with Window size $7 \times 7$ and alpha cut value 0.5100 gives zero MD and zero FA. For the $80 \%$ of impulse noise with Window size $7 \times 7$ and alpha cut value 0.5500 gives zero MD and zero FA. For the $70 \%$ of impulse noise with Window size 7x7 and alpha cut value 0.5980 gives zero MD and zero FA. For the $60 \%$ of impulse noise with Window size $7 \times 7$ and alpha cut value 0.5996 gives zero $\mathrm{MD}$ and zero FA. For the $50 \%$ of impulse noise with Window size 7x7 and alpha cut value 0.5768 gives zero MD and zero FA. Table-IV describes the Computational require to classify the pixel with various window sizes $5 \times 5$ to $11 \mathrm{X} 11$.

$$
P_{7 \times 7}=\left[\begin{array}{lllllll}
255 & 0 & 0 & 126 & 108 & 105 & 0 \\
125 & 0 & 0 & 255 & 107 & 0 & 255 \\
255 & 124 & 186 & 138 & 0 & 255 & 255 \\
135 & 0 & 0 & 255 & 255 & 0 & 255 \\
0 & 0 & 255 & 0 & 150 & 255 & 152 \\
0 & 255 & 255 & 255 & 255 & 255 & 0 \\
0 & 255 & 255 & 255 & 255 & 0 & 127
\end{array}\right]
$$

Fig.1. Input $7 \times 7$ image corrupted with $70 \%$ of salt and pepper noise.

Table 1. Fuzzy entropy of the each pixel

\begin{tabular}{|l|l|}
\hline Gray value & Fuzzy entropy \\
\hline $\mathbf{0}$ & $\mathbf{0 . 9 9 2 4}$ \\
\hline 126 & 0.0332 \\
\hline 108 & 0.1711 \\
\hline 105 & 0.1996 \\
\hline 125 & 0.0387 \\
\hline 107 & 0.1804 \\
\hline 124 & 0.0446 \\
\hline 186 & 0.3870 \\
\hline 138 & 0.0012 \\
\hline 135 & 0.0010 \\
\hline 150 & 0.0523 \\
\hline 152 & 0.0656 \\
\hline 127 & 0.0279 \\
\hline $\mathbf{2 5 5}$ & $\mathbf{0 . 9 4 4 7}$ \\
\hline
\end{tabular}

From that, window size $7 \times 7$ requires average less computational time to classify the pixels with the noise density ranges from $30 \%$ to $90 \%$.

Restoration performance of proposed method implemented using standard gray scale images of size $256 \times 256$ with 8 -bit resolution. Each of the test images is degraded with Noise Model-I and noise density ranging from $10 \%$ to $90 \%$ in $10 \%$ noise step. For comparison, degraded images also restored using the existing filters adaptive median filter (AM), Decision-Based algorithm for Impulse Noise Removal(DBA)[13], DBUTMF[14],NAFSM[11], boundary discriminative noise detection (BDND)[12] and cloud model filter(CM)[16]. AM utilizes the adaptive window mechanism for recognizing.

Corrupted and uncorrupted pixels, next to the filtering approach, was applied, for AMF initial window size is $3 \times 3$ consider and incrementing the window size maximum to 39 with 2 in step.

The DBA is implemented with the $3 \times 3$ window, it removes only corrupted pixel by the median value of its neighboring pixels. For the BDND in the first iteration, the window size $21 \times 21$ is used, if it fails to find the impulse noise, conditionally invokes the second iteration with window size $3 \times 3$ Initial window size of $3 \times 3$ to maximum window size of $13 \times 13$ with 2 step increment is used to implement the CM.For the FEIND filter window of interest, $7 \times 7$ is used for pixel classification.

Table I \& II are show the restoration results of the Lena image corrupted with noise density range from 
$30 \%$ of impulse noise to $80 \%$ impulse noise in terms of PSNR, [27] and NAE. The table values show that the proposed method gives good performance compare to existing filters. In other two images house and parrot the proposed method gives the better results compare to AM, DBA, MDBUTMF and NASFM and gives average performance compare to BDND and CM filters. Fig.3.a shows original Lena and parrot images, Fig.3.b represents the images corrupted with $30 \%$ impulse noise, Fig.3.c represent the noise image with $60 \%$ impulse noise and Fig.3.d represents the noise images with $80 \%$ impulse noise.

Fig.4 to Fig.8 illustrate the visual quality of the restored images of filters DBA, DBUTM, AMF, NAFSM,
BDND, CM and proposed method. Fig. 4 and Fig.8 conclude that all the filters exhibit almost similar visual quality at $30 \%$ noise level, from the Fig.5 and Fig. 8 we conclude that the visual quality of the DBA method is poor at noise level $60 \%$, and proposed method provides the better visual quality compared to remaining state of art algorithms. From the Fig.6 and Fig.9, we conclude that the DBA, MDBUTMF and AMF filters provide the poor visual quality at noise level $80 \%$ and NAFSM, BDND methods provide better visual quality compare to DBA, DBUTMF and AMF filters, CM and proposed methods give best visual quality compared to remaining all the methods.

Table 2. Comparison of Noise Detection Accuracy in MD And FA In 5x5 For Lena Image at Various Alpha Cut Values

\begin{tabular}{|c|c|c|c|c|c|c|c|c|c|c|c|c|c|c|}
\hline \multirow{3}{*}{ Alpha cut values } & \multicolumn{14}{|c|}{ Noise $\%$} \\
\hline & \multicolumn{2}{|c|}{30} & \multicolumn{2}{|c|}{40} & \multicolumn{2}{|c|}{50} & \multicolumn{2}{|c|}{60} & \multicolumn{2}{|c|}{70} & \multicolumn{2}{|c|}{80} & \multicolumn{2}{|c|}{90} \\
\hline & MD & FA & MD & FA & MD & FA & MD & FA & MD & FA & MD & FA & MD & FA \\
\hline 0.2 & 22494 & 0 & 19642 & 0 & 16010 & 0 & 13004 & 0 & 10054 & 0 & 6538 & 0 & 6099 & 0 \\
\hline 0.3 & 13257 & 0 & 11988 & 0 & 10853 & 0 & 9033 & 0 & 7345 & 0 & 4686 & 0 & 5899 & 0 \\
\hline 0.4 & 6575 & 0 & 6415 & 0 & 6284 & 0 & 5751 & 0 & 4919 & 0 & 3197 & 0 & 5677 & 0 \\
\hline 0.5 & 747 & 0 & 1379 & 0 & 2283 & 0 & 2587 & 0 & 488 & 0 & 1379 & 0 & 397 & 0 \\
\hline 0.6 & 0 & 5458 & 0 & 3556 & 0 & 1178 & 46 & 0 & 465 & 0 & 45 & 0 & 0 & 869 \\
\hline 0.7 & 0 & 8177 & 0 & 5991 & 0 & 3045 & 0 & 1654 & 0 & 889 & 0 & 2044 & 0 & 5121 \\
\hline 0.8 & 0 & 7908 & 0 & 7871 & 0 & 5471 & 0 & 3574 & 0 & 4518 & 0 & 9435 & 0 & 17382 \\
\hline 0.9 & 0 & 9795 & 0 & 12470 & 0 & 11247 & 0 & 11457 & 0 & 15818 & 0 & 24333 & 0 & 35975 \\
\hline
\end{tabular}

Table 3. Comparison of Noise Detection Accuracy in MD and FA in 7x7 For Lena Image at Various Alpha Cut Values

\begin{tabular}{|c|c|c|c|c|c|c|c|c|c|c|c|c|c|c|}
\hline \multirow{3}{*}{ Alpha cut values } & \multicolumn{14}{|c|}{ Noise $\%$} \\
\hline & \multicolumn{2}{|c|}{30} & \multicolumn{2}{|c|}{40} & \multicolumn{2}{|c|}{50} & \multicolumn{2}{|c|}{60} & \multicolumn{2}{|c|}{70} & \multicolumn{2}{|c|}{80} & \multicolumn{2}{|c|}{90} \\
\hline & MD & FA & MD & FA & MD & FA & MD & FA & MD & FA & MD & FA & MD & FA \\
\hline 0.2 & 20201 & 0 & 18492 & 0 & 15545 & 0 & 12237 & 0 & 9730 & 0 & 6274 & 0 & 3177 & 0 \\
\hline 0.3 & 11787 & 0 & 11912 & 0 & 10782 & 0 & 8886 & 0 & 6853 & 0 & 4508 & 0 & 2268 & 0 \\
\hline 0.4 & 4855 & 0 & 6194 & 0 & 5917 & 0 & 5577 & 0 & 4701 & 0 & 3172 & 0 & 1621 & 0 \\
\hline 0.5 & 0 & 923 & 778 & 0 & 635 & 0 & 376 & 0 & 149 & 0 & 68 & 0 & 71 & 0 \\
\hline 0.6 & 0 & 5744 & 0 & 3122 & 0 & 523 & 0 & 36 & 243 & 0 & 0 & 422 & 0 & 893 \\
\hline 0.7 & 0 & 9004 & 0 & 5685 & 0 & 698 & 0 & 1400 & 0 & 846 & 0 & 1528 & 0 & 2310 \\
\hline 0.8 & 0 & 11693 & 0 & 9026 & 0 & 2986 & 0 & 2849 & 0 & 2674 & 0 & 5208 & 0 & 11815 \\
\hline 0.9 & 0 & 14098 & 0 & 13745 & 0 & 9894 & 0 & 8578 & 0 & 11038 & 0 & 20988 & 0 & 35156 \\
\hline
\end{tabular}

Table 4. CPU Time In Seconds For Bridge Image

\begin{tabular}{|c|c|c|c|c|c|c|c|}
\hline \multirow{2}{*}{ WINDOWS } & \multicolumn{7}{|c|}{ Noise $\%$} \\
\hline & 30 & 40 & 50 & 60 & 70 & 80 & 90 \\
\hline $5 \times 5$ & 2.148925 & 2.098575 & 2.167425 & 2.109863 & 2.108038 & 2.10855 & 2.118725 \\
\hline $7 X 7$ & 2.913102 & 2.874481 & 3.068724 & 2.937363 & 2.769388 & 2.8508 & 3.025413 \\
\hline $9 \times 9$ & 3.682938 & 4.454363 & 4.9594 & 3.807875 & 3.887388 & 4.046488 & 4.0685 \\
\hline $11 \mathrm{X} 11$ & 5.373723 & 5.495475 & 6.081713 & 5.474063 & 7.592563 & 5.72225 & 5.30525 \\
\hline
\end{tabular}

Table 5. Comparison of Noise Detection Accuracy In MD and FA in 9x9 For Lena Image at Various Alpha Cut Values

\begin{tabular}{|c|c|c|c|c|c|c|c|c|c|c|c|c|c|c|}
\hline \multirow{3}{*}{ Alpha cut values } & \multicolumn{14}{|c|}{ Noise \% } \\
\hline & \multicolumn{2}{|c|}{30} & \multicolumn{2}{|c|}{40} & \multicolumn{2}{|c|}{50} & \multicolumn{2}{|c|}{60} & \multicolumn{2}{|c|}{70} & \multicolumn{2}{|c|}{80} & \multicolumn{2}{|c|}{90} \\
\hline & MD & FA & MD & FA & MD & FA & MD & FA & $\mathrm{MD}$ & FA & MD & FA & MD & FA \\
\hline 0.2 & 20761 & 0 & 18408 & 0 & 15555 & 0 & 12265 & 0 & 9639 & 0 & 6257 & 0 & 3066 & 0 \\
\hline 0.3 & 12907 & 0 & 12525 & 0 & 11189 & 0 & 8947 & 0 & 7066 & 0 & 4635 & 0 & 2256 & 0 \\
\hline 0.4 & 5629 & 0 & 7159 & 0 & 7323 & 0 & 5581 & 0 & 4789 & 0 & 3144 & 0 & 1587 & 0 \\
\hline 0.5 & 0 & 502 & 2065 & 0 & 3222 & 0 & 2384 & 0 & 1793 & 0 & 698 & 0 & 0 & 87 \\
\hline 0.6 & 0 & 5358 & 0 & 2972 & 6969 & 0 & 29 & 0 & 0 & 52 & 0 & 1002 & 0 & 1383 \\
\hline 0.7 & 0 & 8985 & 0 & 5872 & 0 & 125 & 0 & 1079 & 0 & 1094 & 0 & 1768 & 0 & 2227 \\
\hline 0.8 & 0 & 12073 & 0 & 9946 & 0 & 5110 & 0 & 2280 & 0 & 1953 & 0 & 4220 & 0 & 8478 \\
\hline 0.9 & 0 & 14416 & 0 & 14757 & 0 & 10463 & 0 & 7639 & 0 & 9077 & 0 & 18469 & 0 & 34910 \\
\hline
\end{tabular}


Table 6. Comparison of Noise Detection Accuracy In MD And FA in 11x11 For Lena Image at Various Alpha Cut Values

\begin{tabular}{|c|c|c|c|c|c|c|c|c|c|c|c|c|c|c|}
\hline \multirow{3}{*}{ Alpha cut values } & \multicolumn{14}{|c|}{ Noise $\%$} \\
\hline & \multicolumn{2}{|c|}{30} & \multicolumn{2}{|c|}{40} & \multicolumn{2}{|c|}{50} & \multicolumn{2}{|c|}{60} & \multicolumn{2}{|c|}{70} & \multicolumn{2}{|c|}{80} & \multicolumn{2}{|c|}{90} \\
\hline & $\mathrm{MD}$ & FA & $\mathrm{MD}$ & FA & $\mathrm{MD}$ & FA & MD & FA & $\mathrm{MD}$ & FA & MD & FA & MD & FA \\
\hline 0.2 & 21159 & 0 & 19964 & 0 & 16032 & 0 & 12477 & 0 & 9746 & 0 & 6178 & 0 & 3123 & 0 \\
\hline 0.3 & 13756 & 0 & 13377 & 0 & 11785 & 0 & 9335 & 0 & 7191 & 0 & 4415 & 0 & 2476 & 0 \\
\hline 0.4 & 6950 & 0 & 7649 & 0 & 7440 & 0 & 6290 & 0 & 5007 & 0 & 3378 & 0 & 1706 & 0 \\
\hline 0.5 & 304 & 0 & 2685 & 0 & 3284 & 0 & 2149 & 0 & 1851 & 0 & 597 & 0 & 0 & 444 \\
\hline 0.6 & 0 & 4655 & 0 & 1617 & 307 & 0 & 0 & 45 & 0 & 470 & 0 & 1155 & 0 & 1979 \\
\hline 0.7 & 0 & 8750 & 0 & 6253 & 0 & 2026 & 0 & 1199 & 0 & 1649 & 0 & 2212 & 0 & 2741 \\
\hline 0.8 & 0 & 11880 & 0 & 9876 & 0 & 4302 & 0 & 2126 & 0 & 2221 & 0 & 3727 & 0 & 7034 \\
\hline 0.9 & 0 & 14383 & 0 & 14980 & 0 & 9970 & 0 & 7089 & 0 & 8364 & 0 & 17177 & 0 & 35381 \\
\hline
\end{tabular}

Table 7.Comparison Of Zero MD and FA For Lena Image At Different Alpha Cut Values For Various Noise Densities With Window Size 7x7

\begin{tabular}{|c|c|c|c|c|c|c|c|c|c|c|}
\hline \multirow{2}{*}{ Alpha cut values } & \multicolumn{10}{|c|}{ Noise $\%$} \\
\hline & 50 & & 60 & & 70 & & 80 & & 90 & \\
\hline & $\mathrm{MD}$ & FA & MD & $\mathrm{FA}$ & MD & FA & MD & FA & MD & FA \\
\hline 0.5100 & -- & -- & -- & -- & -- & -- & -- & -- & 0 & 0 \\
\hline 0.5500 & -- & -- & -- & -- & -- & -- & 0 & 0 & -- & -- \\
\hline 0.5980 & -- & -- & -- & -- & 0 & 0 & -- & -- & -- & -- \\
\hline 0.5996 & -- & -- & 0 & 0 & -- & -- & -- & -- & -- & -- \\
\hline 0.5768 & 0 & 0 & -- & -- & -- & -- & -- & -- & -- & -- \\
\hline
\end{tabular}

Table 8.Comparison of Noise Detection Accuracy In MD And FA in 7x7 For Bridge Image At Various Alpha Cut Values

\begin{tabular}{|c|c|c|c|c|c|c|c|c|c|c|c|c|c|c|}
\hline \multirow{3}{*}{ Alpha cut values } & \multicolumn{14}{|c|}{ Noise $\%$} \\
\hline & \multicolumn{2}{|c|}{30} & \multicolumn{2}{|c|}{40} & \multicolumn{2}{|c|}{50} & \multicolumn{2}{|c|}{60} & \multicolumn{2}{|c|}{70} & \multicolumn{2}{|c|}{80} & \multicolumn{2}{|c|}{90} \\
\hline & MD & FA & MD & FA & MD & FA & MD & FA & $\mathrm{MD}$ & FA & MD & FA & MD & FA \\
\hline 0.2 & 25078 & 0 & 21744 & 0 & 17911 & 0 & 14207 & 0 & 10735 & 0 & 7027 & 0 & 3608 & 0 \\
\hline 0.3 & 18439 & 0 & 15892 & 0 & 13261 & 0 & 14608 & 0 & 7878 & 0 & 5209 & 0 & 2706 & 0 \\
\hline 0.4 & 11843 & 0 & 10716 & 0 & 9233 & 0 & 7239 & 0 & 5543 & 0 & 3642 & 0 & 1888 & 0 \\
\hline 0.5 & 88 & 0 & 24 & 0 & 61 & 0 & 153 & 0 & 174 & 0 & 241 & 0 & 507 & 0 \\
\hline 0.6 & 0 & 37 & 0 & 09 & $\mathbf{0}$ & 83 & O & 144 & 0 & 172 & 0 & 149 & 0 & 937 \\
\hline 0.7 & 0 & 5275 & 0 & 3885 & 0 & 1334 & 0 & 818 & 0 & 842 & 0 & 1254 & 0 & 2393 \\
\hline 0.8 & 0 & 8842 & 0 & 7467 & 0 & 4971 & 0 & 3036 & 0 & 3825 & 0 & 5897 & 0 & 12464 \\
\hline 0.9 & 0 & 12925 & 0 & 12439 & 0 & 9944 & 0 & 8745 & 0 & 12203 & 0 & 22636 & 0 & 36144 \\
\hline
\end{tabular}

Table 9. Comparison Of Restoration Results For 'Leena' Test Image In Psnr (Db) And NAE

\begin{tabular}{|c|c|c|c|c|c|c|c|c|c|c|}
\hline \multirow{2}{*}{ Methods } & \multirow[b]{2}{*}{$30 \%$} & \multicolumn{4}{|c|}{ PSNR(dB) } & \multicolumn{5}{|c|}{ NAE } \\
\hline & & $40 \%$ & $50 \%$ & $60 \%$ & $80 \%$ & $20 \%$ & $40 \%$ & $50 \%$ & $60 \%$ & $80 \%$ \\
\hline $\mathrm{AM}$ & 35.6125 & 33.6430 & 32.6876 & 30.7285 & 28.8328 & 0.82253 & 0.81653 & 0.7184 & 0.7119 & 0.6103 \\
\hline DBA & 28.8834 & 18.8552 & 15.5678 & 12.2098 & 8.1785 & 0.9375 & 0.9225 & 0.9115 & 0.8618 & 0.6919 \\
\hline MDBUTMF & 34.2395 & 30.2826 & 28.5198 & 26.1650 & 19.3849 & 0.9689 & 0.9243 & 0.8864 & 0.8093 & 0.4187 \\
\hline NAFSM & 28.5933 & 23.6392 & 22.5576 & 21.5733 & 18.9354 & 0.8467 & 0.8258 & 0.7023 & 0.7047 & 0.6987 \\
\hline BDND & 36.6334 & 33.6543 & 30.9698 & 28.2745 & 24.6634 & 0.9748 & 0.9601 & 0.9466 & 0.9345 & 0.8663 \\
\hline $\mathrm{CM}$ & 38.4832 & 34.2883 & 32.5209 & 30.8287 & 27.6889 & 0.9732 & 0.9510 & 0.9498 & 0.9137 & 0.8789 \\
\hline PROPOSED & 39.2674 & 36.0177 & 33.4274 & 32.7657 & 29.2326 & 0.9848 & 0.9610 & 0.9510 & 0.9232 & 0.8879 \\
\hline
\end{tabular}

Table10. Comparison Of Restoration Results For 'House' Test Image In PSNR (Db) and NAE

\begin{tabular}{|c|c|c|c|c|c|c|c|c|c|c|}
\hline \multirow{2}{*}{ Methods } & & \multicolumn{4}{|c|}{ PSNR(dB) } & \multicolumn{5}{|c|}{ NAE } \\
\hline & $30 \%$ & $40 \%$ & $50 \%$ & $60 \%$ & $80 \%$ & $20 \%$ & $40 \%$ & $50 \%$ & $60 \%$ & $80 \%$ \\
\hline $\mathrm{AM}$ & 32.1074 & 30.1365 & 28.1808 & 27.1863 & 26.5904 & 0.8234 & 0.7199 & 0.6108 & 0.6042 & 0.5842 \\
\hline DBA & 26.8497 & 18.1944 & 15.0307 & 12.2714 & 8.14053 & 0.8057 & 0.6107 & 0.5225 & 0.5052 & 0.4287 \\
\hline MDBUTMF & 39.0911 & 34.2661 & 32.0790 & 28.7031 & 20.2540 & 0.9669 & 0.9242 & 0.8851 & 0.7933 & 0.3729 \\
\hline NAFSM & 26.2650 & 26.4591 & 26.5212 & 26.7030 & 27.2948 & 0.7705 & 0.6990 & 0.5558 & 0.5056 & 0.4014 \\
\hline BDND & 39.9874 & 37.1459 & 35.8447 & 32.9685 & 30.5698 & 0.9789 & 0.9698 & 0.9124 & 0.87495 & 0.8024 \\
\hline $\mathrm{CM}$ & 40.8965 & 38.3698 & 36.4589 & 33.4478 & 32.4521 & 0.9801 & 0.9756 & 0.9678 & 0.95123 & 0.8147 \\
\hline PROPOSED & 38.9439 & 35.0503 & 33.2855 & 31.5900 & 27.5910 & 0.9876 & 0.9742 & 0.9601 & 0.95295 & 0.8265 \\
\hline
\end{tabular}


Table 11. Comparison Of Restoration Results For 'Parrot' Test Image In Psnr (Db) And Mae

\begin{tabular}{|c|c|c|c|c|c|c|c|c|c|c|}
\hline \multirow{2}{*}{ Methods } & & \multicolumn{4}{|c|}{ PSNR } & \multicolumn{5}{|c|}{ SSIM } \\
\hline & $30 \%$ & $40 \%$ & $\mathbf{5 0 \%}$ & $60 \%$ & $80 \%$ & $20 \%$ & $40 \%$ & $\mathbf{5 0 \%}$ & $60 \%$ & $80 \%$ \\
\hline $\mathrm{AM}$ & 32.4287 & 36.4892 & 30.4763 & 27.4998 & 25.7119 & 0.1317 & 0.1284 & 0.1210 & 0.1243 & 0.1124 \\
\hline DBA & 25.7226 & 17.8397 & 14.2937 & 11.7089 & 7.53686 & 0.7705 & 0.4431 & 0.2428 & 0.1325 & 0.0343 \\
\hline MDBUTMF & 34.5077 & 29.6318 & 27.2146 & 24.3617 & 17.2021 & 0.9577 & 0.8995 & 0.8418 & 0.7469 & 0.3360 \\
\hline NAFSM & 25.3554 & 25.4609 & 25.4866 & 25.7657 & 26.6167 & 0.3527 & 0.3520 & 0.3527 & 0.3510 & 0.3269 \\
\hline BDND & 36.2255 & 34.1117 & 30.4456 & 28.9633 & 26.1123 & 0.9654 & 0.8963 & 0.8678 & 0.7891 & 0.5669 \\
\hline $\mathrm{CM}$ & 38.2566 & 36.1996 & 33.7789 & 32.1455 & 30.7788 & 0.9755 & 0.9214 & 0.9012 & 0.8852 & 0.7960 \\
\hline PROPOSED & 34.9120 & 31.0264 & 29.7323 & 28.3504 & 24.7187 & 0.9624 & 0.9188 & 0.8932 & 0.8624 & 0.7733 \\
\hline
\end{tabular}

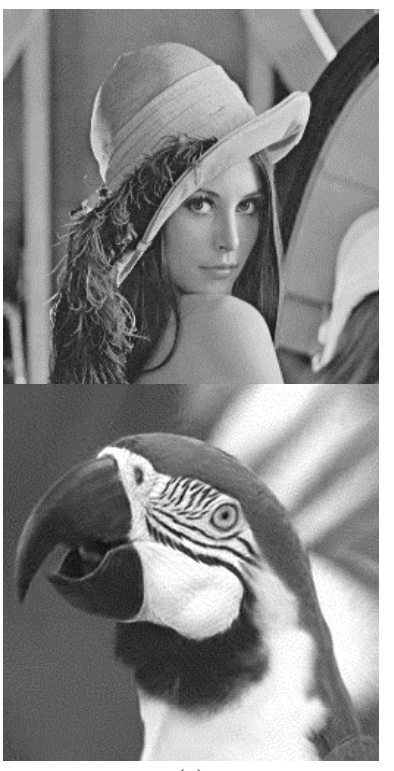

(a)

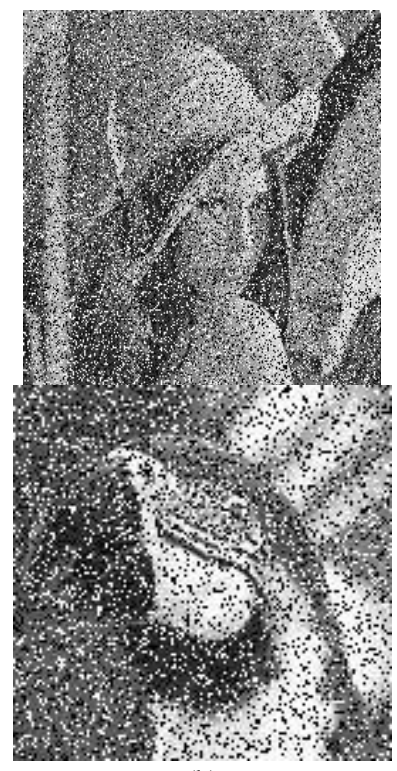

(b)

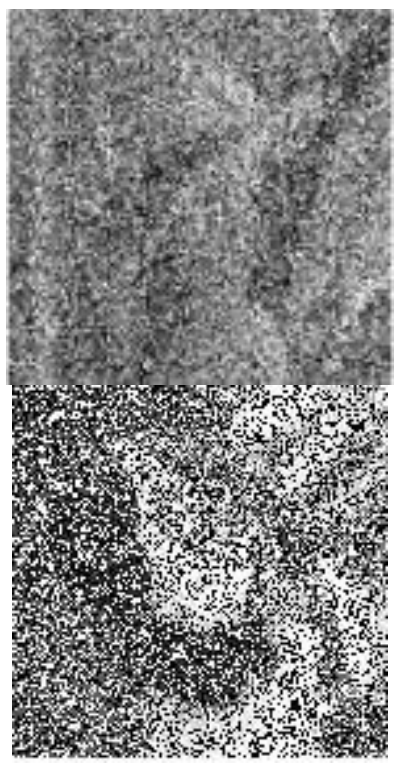

(c)

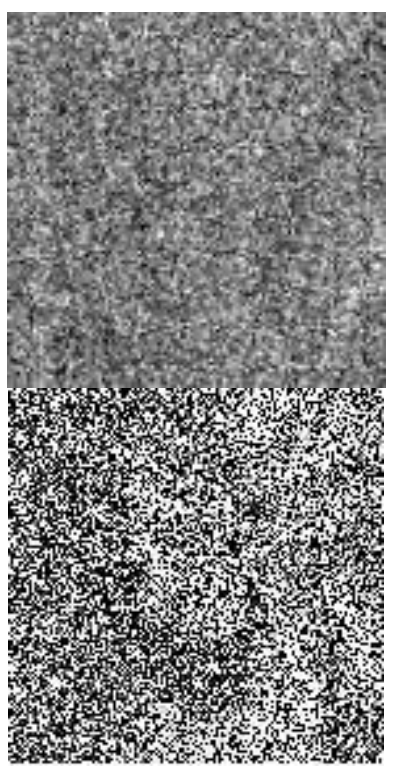

(d)

Fig.3.a) Original images b)Images corrupted with $30 \%$ noise c) Images corrupted with $60 \%$ noise d) Images corrupted with $80 \%$ noise

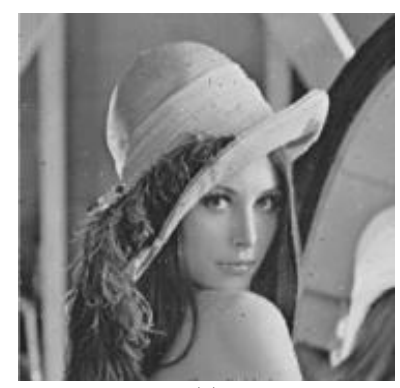

(a)

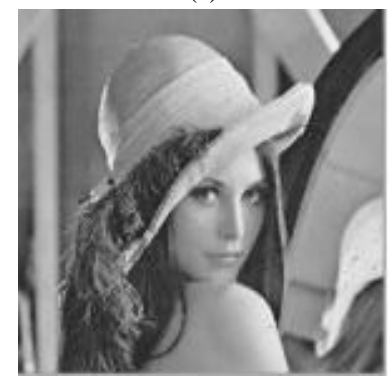

(e)

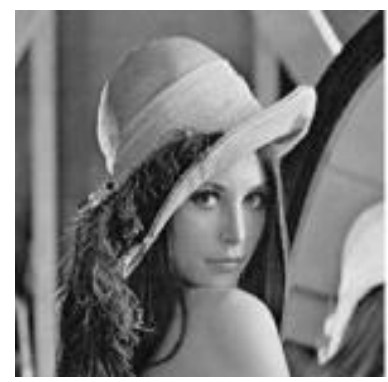

(b)

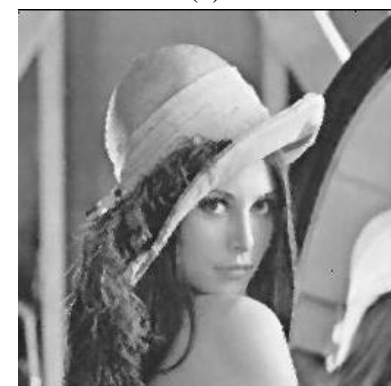

(f)

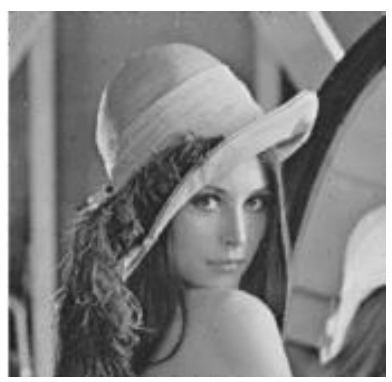

(c)

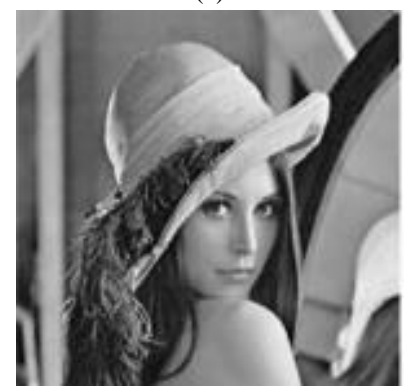

(g)

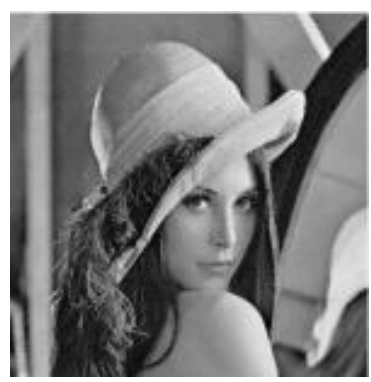

(d)

Fig.4. Results of denoising corrupted image "Lena," with 30\% impulse noise density (a)DBA (b)MDBUTMF (c)AMF(d)NAFSM(e)BDND(f)CM And (g)FEIND 


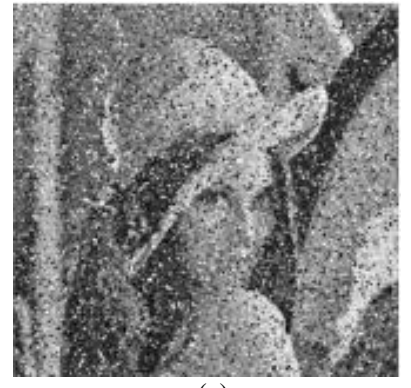

(a)

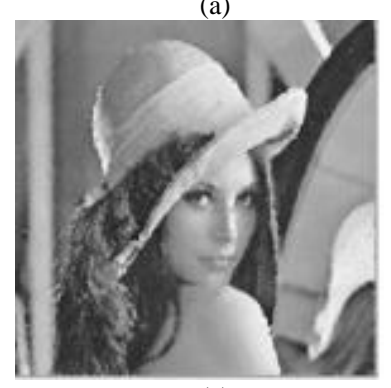

(e)

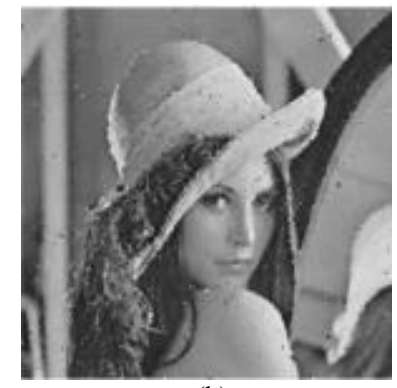

(b)

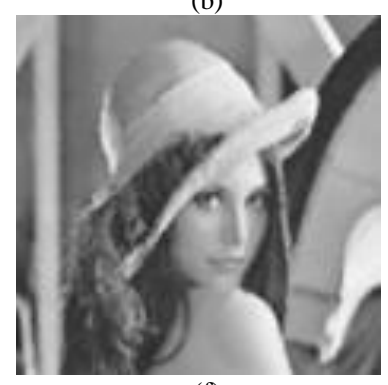

(f)

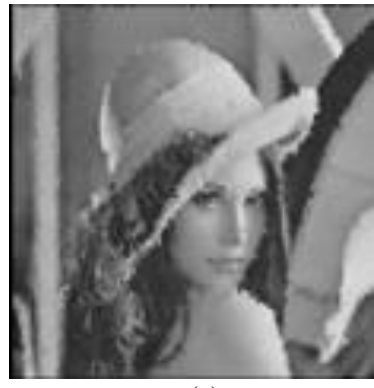

(c)

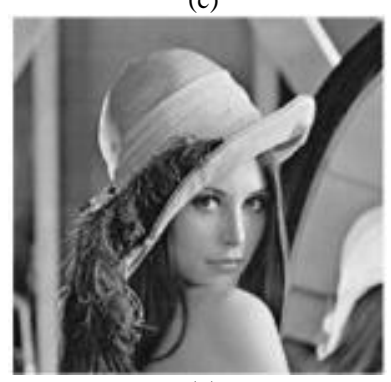

(g)

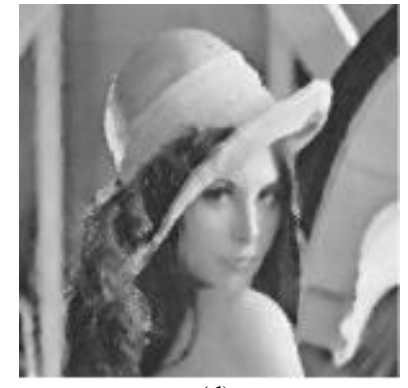

(d)

Fig.5. Results of denoising corrupted image "Lena," with 60\% impulse noise density (a) DBA (b) MDBUTMF (c) AMF (d) NAFSM (e) BDND (f) CM and (g) FEIND

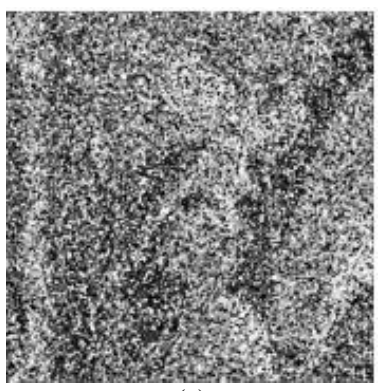

(a)

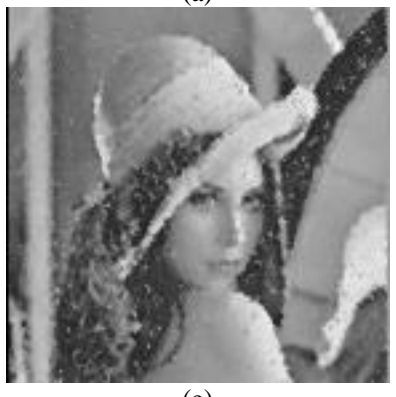

(e)

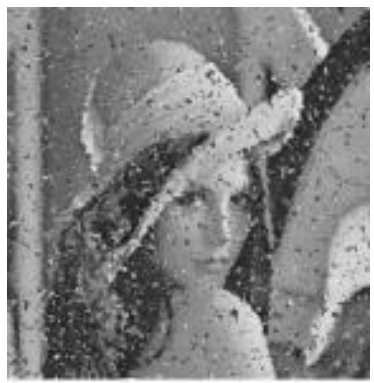

(b)

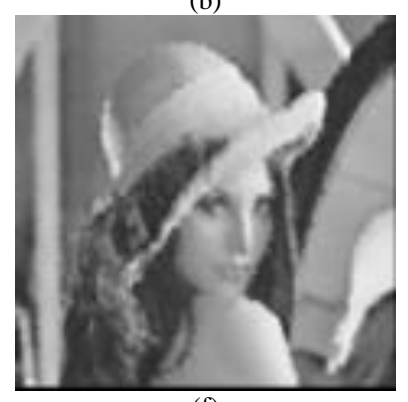

(f)

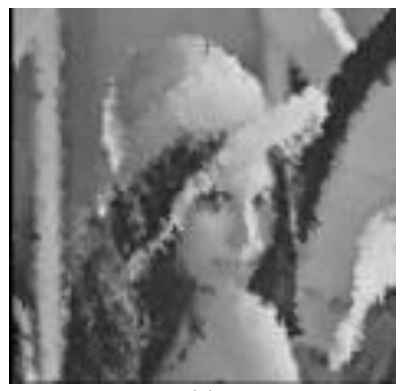

(c)

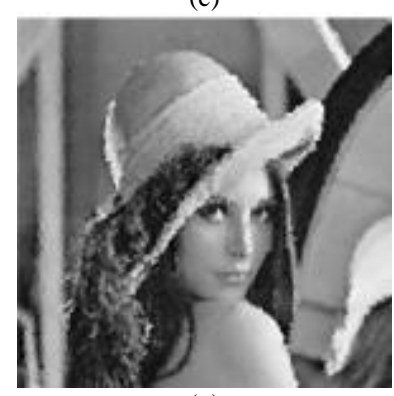

(g)

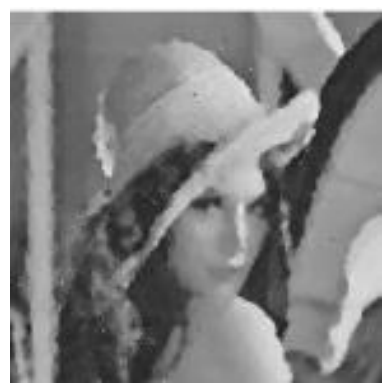

(d)

Fig.6. Results of denoising corrupted image "Lena," with $80 \%$ impulse noise density (a) DBA (b) MDBUTMF (c) AMF (d) NAFSM (e) BDND (f) CM and (g) FEIND

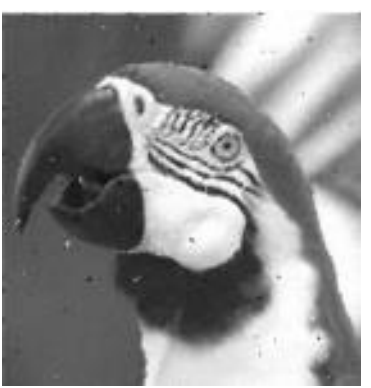

(a)

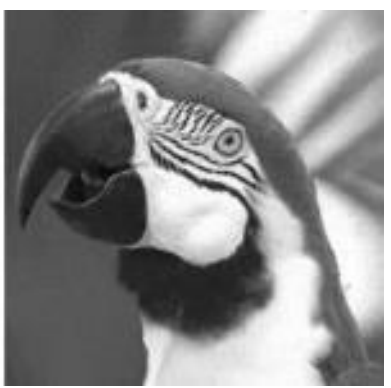

(b)

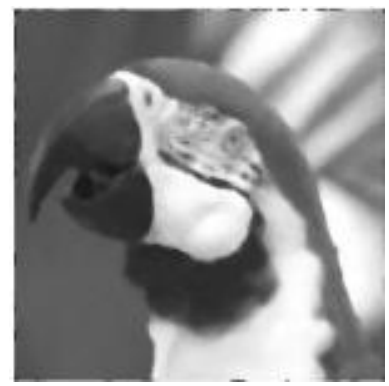

(c)

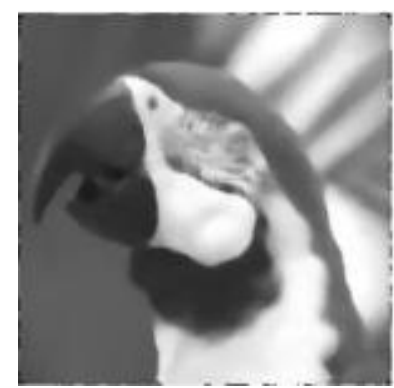

(d) 


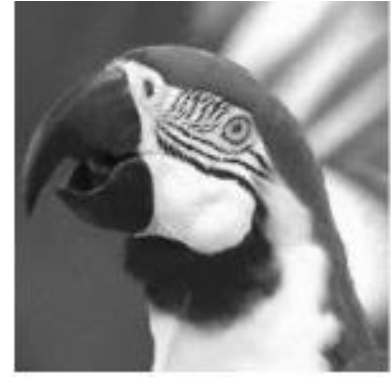

(e)

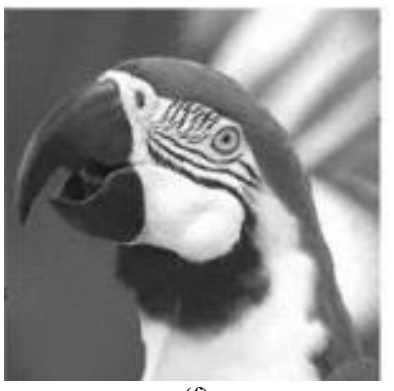

(f)

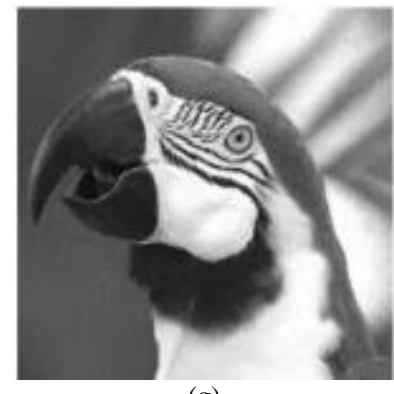

(g)

Fig.7. Results of denoising corrupted image "Parrot," with 30\% impulse noise density (a) DBA (b) MDBUTMF (c) AMF (d) NAFSM (e) BDND (f) $\mathrm{CM}$ and (g) FEIND

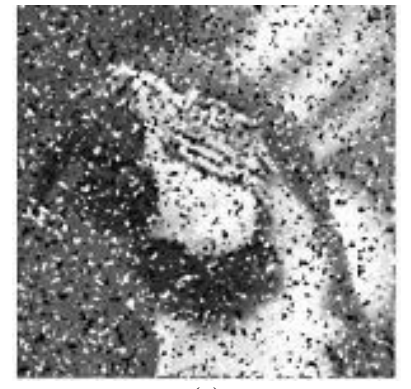

(a)

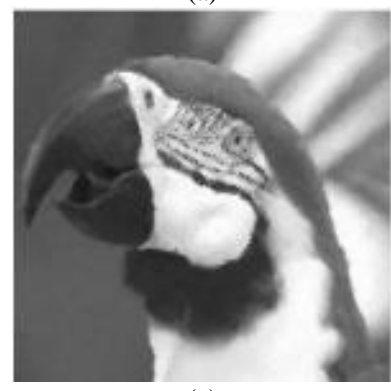

(e)

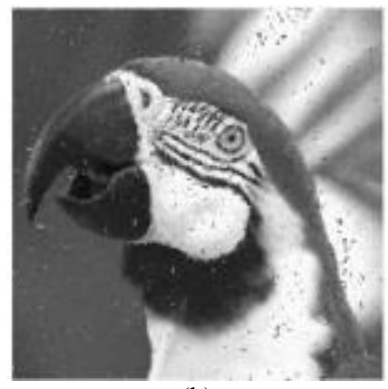

(b)

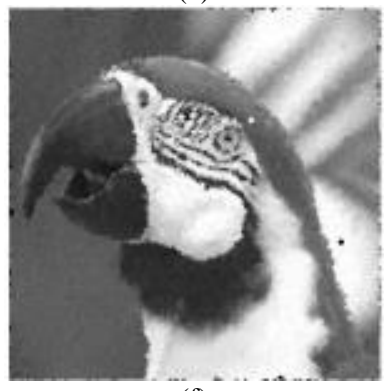

(f)

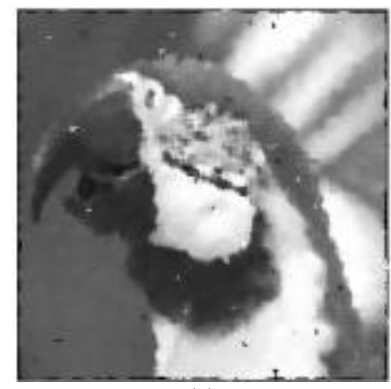

(c)

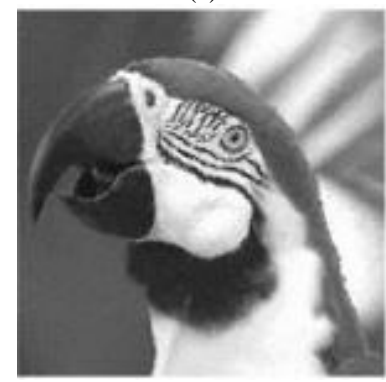

(g)

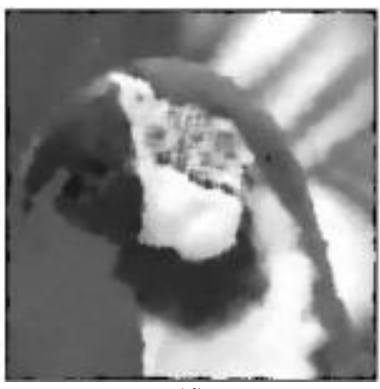

(d)

Fig.8. Results of denoising corrupted image "Parrot," with $60 \%$ impulse noise density (a)DBA (b)MDBUTMF (c)AMF(d)NAFSM(e)BDND(f)CM and (g) FEIND

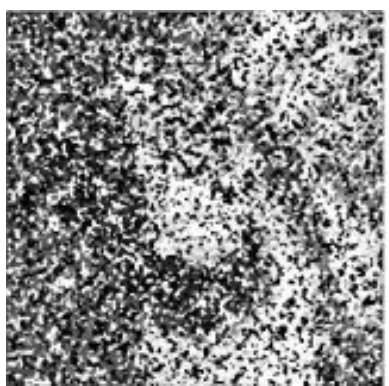

(a)

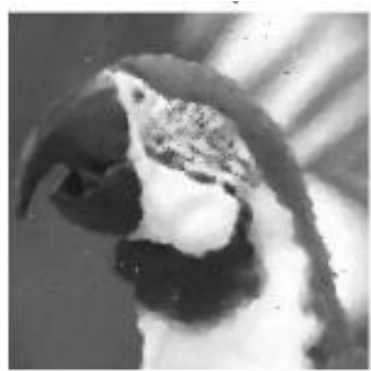

(e)

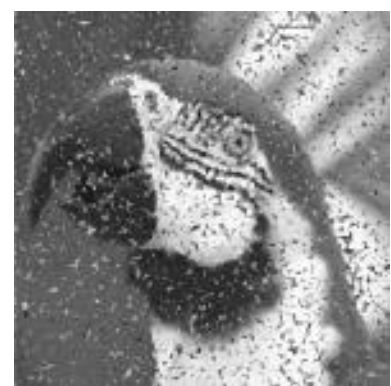

(b)

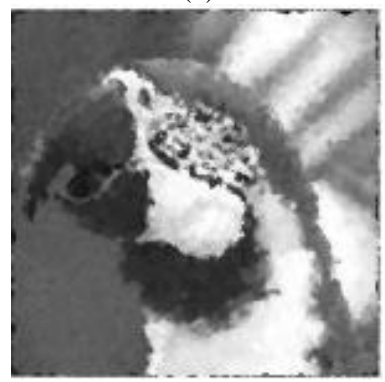

(f)

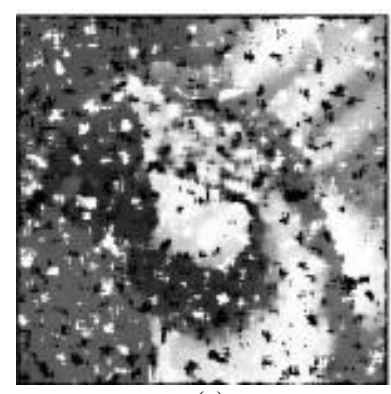

(c)

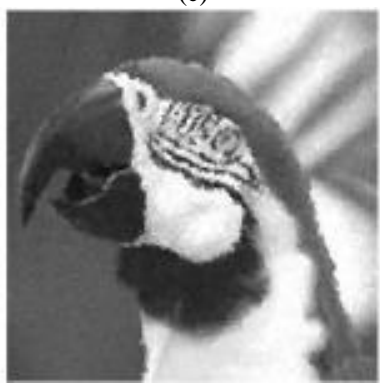

(g)

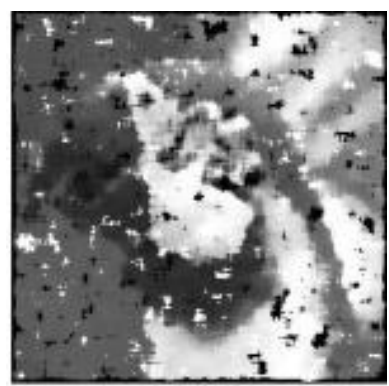

(d)

Fig.9. Results of denoising corrupted image "Parrot," with 80\% impulse noise density (a) DBA (b) MDBUTMF (c) AMF (d) NAFSM (e) BDND (f) CM and (g) FEIND 


\section{CONCLUSIONS}

In this paper, a novel filter with fuzzy entropy for impulse noise detection and removal has been proposed. It represents the uncertainties of the noise perfectly by using the fuzzy entropy, which is helpful in detecting and removing the noise. The experimental results show the FEIND filter is the good among the tested filters, compared with the traditional switching filters. No matter whether, in noise detection, the image details preservation or computational complexity, the FEIND filter makes a good improvement and has the higher performances. Even if the noise level closes to $90 \%$, the texture, the details, and the edges of the images restored by the FEIND filter are preserved with good visual effect. FEIND is not giving the positive results for low impulse noise levels below the $30 \%$ we will address this problem in next paper.

\section{REFERENCES}

[1] RC Gonzalez and RE Woods, "the processing of digital images," Prentice Hall, 2002.

[2] A. I. pitas and Venetsanopou nonlinear, digital filters: Principles and Applications. Norwell, MA: Kluwer, 1990.

[3] J. Astola and P. Kuosmanen, non-linear digital filter concepts. Boca Raton, FL: CRC 1997.

[4] T. Sun and Y. Neuvo, "Detail preservation of median filters in the image processing," Recognit.Lett., 15, pp. 341-347 1994.

[5] D. Florencio and R. Schafer, "the inauguration median filter using local statistics signal," in Proc. SPIE Int. Symp. The image processing, visual communication, Chicago in September 1994.

[6] DRK Brownrigg, "the weighted median filter" ACM Commun., 27, No. 8, pp. 807-818, August 1984.

[7] Ko SJ and YH Lee, "center weighted median filters and their applications to improve the image," IEEE Trans. Syst. Circuits, vol. 38, no. 9, pp. 984-993, September 1991.

[8] H. Hwang and RA Haddad, "Adaptive Median Filters: new algorithms and results," IEEE Transactions on Image Processing, Vol.4, No. 4, April 1995.

[9] DZ Wang and Zhang, "progressive switching median filter for the removal of impulse noise highly corrupted images," IEEE Trans Circuits. Syst. II, processing the analog signal. Figures. 46, no. 1, pp. 78-80, January 1999.

[10] S. Zhang and A. Karim, "A new pulse detector for switching median filters," IEEE Signal Process. Lett., 9, No. 11, pp. 360-363, November The year 2002.

[11] How-Lung Eng and Kai-KuangMa,"Noise adaptive softswitching median filter", IEEE Transactions on Image Processing, Vol.10, No 2, August 2002.

[12] EP Ng KK and Ma, "A switching median filter with boundary discriminative noise detection for extremely corrupted images," IEEE Transactions on Image Processing, 15, No. 6, pp. 1506 - 15162006.

[13] KS Srinivasan and D. Ebenezer "a new fast and efficient decision based removal of high density impulse noise algorithm," IEEE Signal Processing Lett, 14, No. 3, pp.189. - 192, 2007.

[14] S.Esakkirajan,T.V.Kumar,Adabala .Subramanyam And C.H.Prechand "Removal Of High Density Salt And Pepper Noise Through Modified Decision Based
Unsymmetric Trimmed Median Filter" Ieee Signal Processing Letters, Vol. 18, No. 5, May 2011.

[15] Y. Li DY and Artificial Intelligence of the uncertainty, 2007: CRC Press.

[16] Zhe Zhou, "Cognition and removal of impulse noise with uncertainty," IEEE Transactions on Image processing, 21, No. 7, pp. 3157-3167, July 2012.

[17] A. De Luca, S. Termini, "A definition of non-probabilistic entropy in the setting of fuzzy set theory," Information and Control, vol.20, no.4, pp. 301-312, 1972.

[18] G.J. Klir, T.A. Folger, Fuzzy Sets, Uncertainty, and Information, in: Uncertainty and Information, PrenticeHall International Editions, 1988.

[19] L. Xuecheng, "Entropy, distance measure and similarity measure of fuzzy sets and their relations", Fuzzy Sets and Systems, vol. 52, pp. 305-318, 1992.

[20] D. Bhandari and N.R. Pal, "Some new information measure of fuzzy sets", Inform. Sci. vol. 67, pp. 209-228, 1993

[21] H. T Nguyen, "Fuzzy sets and probability", Fuzzy Sets and Systems, vol. 90, pp. 129-132, 1997

[22] H.-M. Lee, C.-M. Chen, J.-M. Chen, and Y.-L. Jou, “An efficient fuzzy classifier with feature selection based on fuzzy entropy," IEEE Trans. On Systems, Man, and Cybernetics-part B: Cybernetics, Vol. 31, No. 3, 2001, pp. 426-432.

[23] SemaKocKayhan, "A phase 2 method to remove impulse noise in images," J. VisCommun. ImageR, Vol 25, pp.478-486, 2014.

[24] PS Windyga "fast, suppression of impulsive noise," IEEE Trans. Process the image, Vol 10, no. 1, pp. 173-179, January 2001.

[25] I. and C. Butakoff Aizenberg, "effective pulse based on sensors sorting criteria," IEEE Signal Process. Lett., 11, No. 3, pp. 363-366, March 2004.

[26] Vijaya Kumar and C.Nagaraju," Identifying and Removal of Impulse Noise with Fuzzy Certainty Degree" IEEE international Conference on Communications and Electronics systems, 2016

[27] Wu Qiu,Feng xiao,Xin Yang,Xuming Zhang,Ming Yuchi,Mingyue Ding,"Research on Fuzzy Enhancement in the Diagnosis of Liver Tumor from B-mode Ultrasound Images", IJIGSP, vol.3, no.3, pp.10-16, 2011.

[28] K. Kannan,"A new Decision Based Median Filter using Cloud Model for the removal of high density Salt and Pepper noise in digital color images", IJIGSP, vol.6, no.4, pp.46-53, 2014

[29] Hani M. Ibrahem,"An Efficient Switching Filter Based on Cubic B-Spline for Removal of Salt-and-Pepper Noise", IJIGSP, vol.6, no.5, pp.45-52, 2014.

[30] Rupinder Kaur, Raman Maini,"Performance Evaluation and Comparative Analysis of Different Filters for Noise Reduction", International Journal of Image, Graphics and Signal Processing (IJIGSP), Vol.8, No.7, pp.9-21, 2016.

\section{Authors' Profiles}

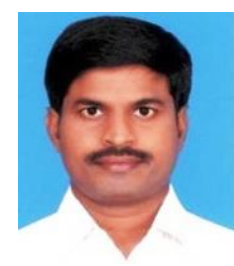

S.Vijaya Kumar Received the B.Tech (CSE) degree from KSRM College of Engineering in 2004. He received the M.Tech (CS) degree from RGMCET Nandyal in 2007. At present pursuing Ph.D. in Digital Image Processing from JNTU Hyderabad and working as Assistant Professor in the department of IT at 
RGMCET, Nandyal. He has got 10 years of teaching experience. His research interest includes Image Processing, Pattern Recognition. He has 14 research papers in various national and international journal and conferences. He has 12 seminars and workshops. He is member of various professional societies like IEEE, IAENG, IACSIT and CSI Email:svksr105@gmail.com

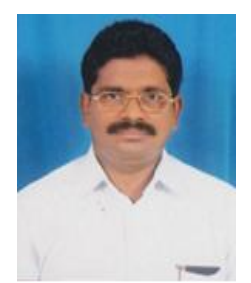

Dr. C. NAGARAJU is currently working as Associate Professor and Head of the Department of Computer Science and Engineering at YSR Engineering College of Yogivemana University, Proddatur, Kadapa District, and Andhra Pradesh, India. He received his B.Tech Degree in Computer Science and Engineering from J.N.T.University,Anantapur, and M.Tech Degree in Computer
Science from J. N. T. University Hyderabad and $\mathrm{PhD}$ in digital Image processing from J. N. T. University Hyderabad. He has got 20 years of teaching experience. He received research excellence award, teaching excellence award and Rayalaseemavidhyaratna award for his credit. He wrote text book on Pattern Recognition Pearson publications. He has six $\mathrm{PhD}$ scholars. He has published seventy seven research papers in various National and International Journals and about thirty research papers in various National and International Conferences. He has attended twenty seminars and workshops. $\mathrm{He}$ delivered 10 keynote addresses. He is member of various professional societies like IEEE, ISTE and CSI. Email:cnrcse@yahoo.com

How to cite this paper: S.Vijaya Kumar, C.Nagaraju," Fuzzy Entropy based Impulse Noise Detection and Correction Method for Digital Images", International Journal of Image, Graphics and Signal Processing(IJIGSP), Vol.10, No.3, pp. 36-46, 2018.DOI: 10.5815/ijigsp.2018.03.05 